\title{
AN FPGA IMPLEMENTATION OF PATTERN-SELECTIVE PYRAMIDAL IMAGE FUSION
}

\author{
Oliver Sims * \\ Institute for System Level Integration \\ Alba Campus \\ Livingston, Scotland, UK \\ oliver.sims@sli-institute.ac.uk
}

\author{
James Irvine \\ Department of Electrical \& Electronic \\ Engineering \\ University of Strathclyde \\ Glasgow, Scotland, UK
}

\begin{abstract}
The aim of image fusion is to combine multiple images (from one or more sensors) into a single composite image that retains all useful data without introducing artefacts. Pattern-selective techniques attempt to identify and extract whole features in the source images to use in the composite. These techniques usually rely on multiresolution image representations such as Gaussian pyramids, which are localised in both the spatial and spatial-frequency domains, since they enable identification of features at many scales simultaneously. This paper presents an FPGA implementation of pyramidal decomposition and subsequent fusion of dual video streams. This is the first reported instance of a hardware implementation of pattern-selective pyramidal image fusion. Use of FPGA technology has enabled a design that can fuse dual video streams (greyscale VGA, 30fps) in real-time, and provides approximately 100 times speedup over a $2.8 \mathrm{GHz}$ Pentium- 4 .
\end{abstract}

\section{INTRODUCTION}

Image fusion allows multiple observations of a scene to be combined, in order to increase the information content presented in a single image, and make the image more effective for its intended application. The images to be fused may originate from a single sensor, perhaps taken with different points of focus, or from multiple sensors that are sensitive to different spectral regions. Image fusion has been used widely in medical, manufacturing, military, and security applications, amongst others [1]. One modern example where image fusion techniques are proving useful is in the detection of concealed weapons by using a composite of thermal and visible-range observations [2]. There are several methods of performing image fusion, with a successful implementation being one that retains all useful information from the source images into a single composite image, without introducing artefacts. Basic methods take no account of

\footnotetext{
*Sponsored by Thales Optronics and EPSRC under the Engineering Doctorate (EngD) programme
}

the image content and perform simple merging of the image data, for instance averaging. More sophisticated methods work at a higher level by identifying detail in the source images and using a selection process to determine the elements that will be used in the final composite.

Several key methods in image fusion rely on the multiscale image pyramid [3]. Image pyramids are a decomposition of a single image into a series of images of varying resolutions, with each image containing data representative of detail at a particular scale. The advantages of a multiscale representation lie in its localisation in both spatial and spatial-frequency domains. The fusion algorithm presented here uses pyramids that have been further decomposed into orientation specific pyramids [4], [5], [6]. It uses simple edge filters (gradient filters) to identify details in the source images along four orientations. These edges are compared, and the most useful features are selected according to some measure of saliency and then carried forward into the composite image. Using gradient pyramids has been found to reduce the artefacts that other pyramidal methods can introduce [4]. The pattern-selective fusion algorithm is complicated and requires thousands of calculations to be performed in order to produce a single output image. For this reason a microprocessor implementation is inherently slow, and real-time processing unfeasible. However, like many image processing algorithms, there are opportunities to exploit parallelism in the algorithm's operation that make an FPGA implementation an attractive option.

This paper describes an implementation of a patternselective fusion algorithm on a single Virtex-2 FPGA. The design uses several novel approaches to enable dual greyscale VGA video streams to be fused in real-time. Note that the source images are assumed to be pre-aligned, and automatic registration tecniques have not been considered here.

\section{PYRAMID GENERATION}

The Gaussian or low pass pyramid and Laplacian bandpass pyramids were introduced by Burt in 1983 [7]. These methods have since been used in a wide variety of applications 
besides fusion, and were a precursor to the development of more general multiresolution methods, in particular the study of wavelets.

The image to be decomposed forms the bottom level of a notional pyramid. Each subsequent higher level of the pyramid is formed by low-pass filtering, and then subsampling by a factor of two, the pyramid level beneath it. The act of low-pass filtering reduces the band limit by one octave, and hence, according to the sampling theorem, subsampling can take place without any loss of information. In reality the generating filter is not "ideal", which means that the following subsampling may result in aliasing; however these effects are usually disregarded for these purposes [8]. The low-pass filter is usually chosen to be a $5 \times 5$ Gaussian, which has the added advantage of being separable.

Hence, each level of the pyramid is produced as

$$
[t] G_{k}=\sum_{m=-2}^{2} \sum_{n=-2}^{2} w(m, n) G_{k-1}(2 i+m, 2 j+n)
$$

for $\mathrm{k}=1, \ldots, \mathrm{N} ; G_{0} \equiv \mathrm{I}$, the original image; w is the filter kernel. This process is usually referred to as REDUCE, when considering the $2 \mathrm{D}$ image as a whole:

$$
G_{k}=\operatorname{REDUCE}\left(G_{k-1}\right)
$$

Since each image is half the size in each dimension of the image below it, it consists of one quarter the number of pixels.

The alternative pyramid type is known as the Laplacian. This is formed as a bandpass pyramid rather than a low pass, and is obtained by subtracting a level of the Gaussian pyramid from the level directly beneath it. Each level of the Laplacian pyramid can thus be thought of as a difference image between two corresponding levels of the Gaussian pyramid. Because two levels of the Gaussian pyramid are different sizes, in order to subtract one from another the resolution of the image at level $\mathrm{k}+1$ must first be increased to the resolution of the image at level $\mathrm{k}$. In order to do this we use the EXPAND operation, which involves upsampling of the smaller image (by inserting zeros), and then interpolation of the missing values by a further application of the generating filter kernel. Then:

$$
L_{k}=G_{k}-\operatorname{EXPAND}\left(G_{k+1}\right)
$$

There must be one fewer levels in the Laplacian pyramid than in the Gaussian. Typically the Gaussian and Laplacian pyramids are generated with three levels above the base image. At levels higher than this the resulting images may become too small to be useful, and the edge effects of successive convolutions with the generating kernel become detrimental. It is possible to perform fusion with less than four pyramid levels, but the ability of the algorithm to distinguish features of different sizes is compromised.

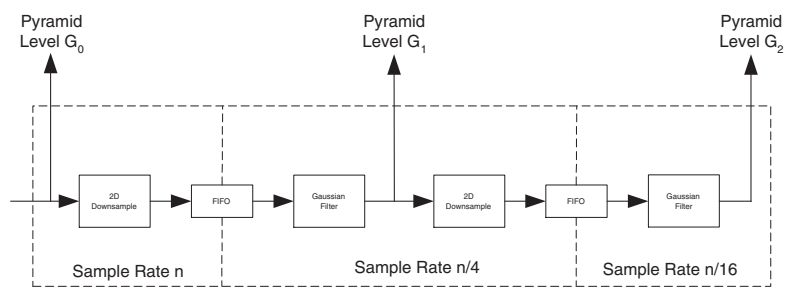

Fig. 1. Block diagram of hardware image pyramid generation.

The Gaussian pyramid is the one used in the process of extracting detail from the source images to be used for fusion. The Laplacian pyramid and a variant of it known as the FSD Laplacian [9], are used in the inverse pyramid transform to reconstruct the composite image.

\subsection{Hardware Implementation}

A block diagram of the image pyramid generation system with two pyramid levels above the base is shown in Fig. 1. This is a multirate design that allows multiple pyramid levels to be generated concurrently. The downsampling stages work by simply discarding certain data values: horizontal downsampling occurs by discarding every other sample; vertical downsampling occurs by discarding every other row. The samples that are not discarded are stored in a FIFO that acts as a buffer between pyramid levels. The next pyramid level operates at a clock rate that is one quarter that of the level below it, to match the fact that it is receiving one quarter the number of samples. In this way the higher level can run concurrently with the lower level, without emptying the FIFO. This structure is repeated for each level of the pyramid, with each level operating at one quarter the clock speed of the level below it. The ability to generate pyramid levels concurrently counteracts the negative effect on performance caused by running portions of the design at slower clock speeds. By using this approach the time to generate the whole pyramid is only 1.1 times that needed to read a full frame of data, compared to upwards of 1.8 times for designs that generate levels sequentially.

\section{DETAIL EXTRACTION AND FUSION}

To extract detail from the levels of the source pyramids four gradient operators are each applied to each level of the source pyramids via a simple convolution. The operators represent derivatives in the horizontal, vertical, and two diagonal directions, and essentially act as edge detection filters 


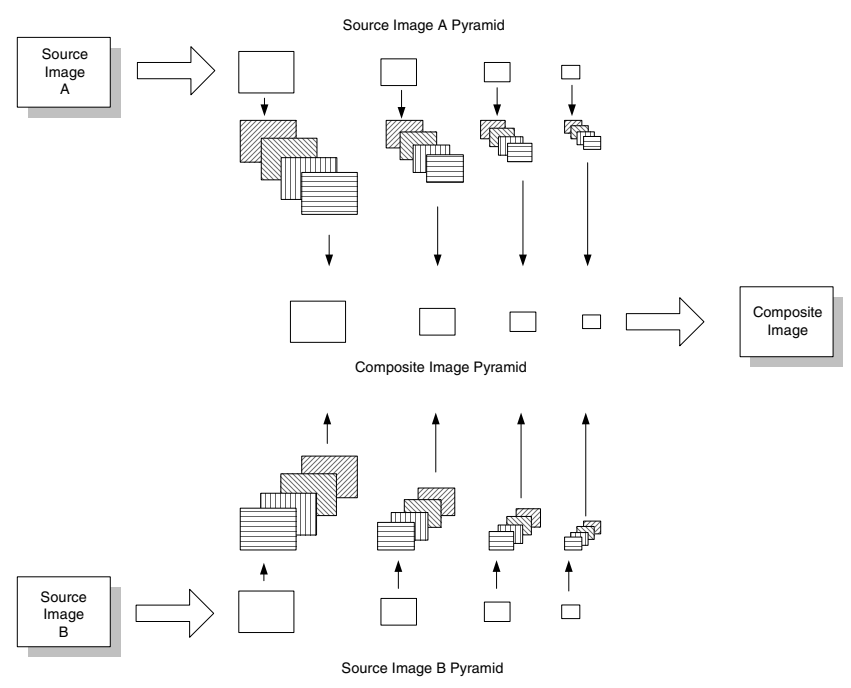

Fig. 2. Gradient pyramid decomposition and fusion

in the four orientations. The gradient filters are as follows:

$$
\begin{aligned}
& d_{1}=\left[\begin{array}{cc}
1 & -1
\end{array}\right] \\
& d_{2}=\left[\begin{array}{cc}
0 & -1 \\
1 & 0
\end{array}\right] \frac{1}{\sqrt{2}} \\
& d_{3}=\left[\begin{array}{c}
-1 \\
1
\end{array}\right] \\
& d_{4}=\left[\begin{array}{cc}
-1 & 0 \\
0 & 1
\end{array}\right] \frac{1}{\sqrt{2}}
\end{aligned}
$$

The resulting set of images is known as a gradient pyramid [5], and can completely represent the original image [10]. The gradient pyramid is essentially a set of gradient maps of the source images at varying scales. As illustrated in Fig. 2, the gradient pyramids constitute a large amount of intermediate data: each level of the two source pyramids is now represented by four gradient maps. Another way to conceptualise this is that the two source pyramids have now been decomposed into four further pyramids each, giving a total of eight full image pyramids to be handled and processed concurrently. Obviously, the ability to work with this amount of data on chip is one of the ways in which an FPGA may achieve large performance gains over a microprocessor based implementation.

Fusion of the gradient pyramids takes place by selecting the most prominent detail from each level. In this application the elements with the greatest absolute value are chosen through a simple comparison, this is an implementation of the simple measure of saliency given in [4]. Other, more complex measures of saliency (also known as ActivityLevel Measurements [11]), based on texture criteria and other higher order attributes, may give better results in some specific circumstances, but the amplitude based measure has been shown to provide good results in the general case [4].

\section{IMAGE RECONSTRUCTION}

Before the inverse transform can take place it is necessary to adjust the format of the composite image pyramid, as the inverse pyramid transform relies on the Laplacian rather than the Gaussian pyramid. The conversion requires a secondary application of the gradient filters, followed by summation and scaling; for a full description of the process see [5]. The output of this process is a composite Laplacian image pyramid that may be inverse transformed. The method of reconstructing an image from its Laplacian pyramid uses the EXPAND operation defined earlier. The starting point for the inverse transform is the top level of the Gaussian pyramid (in this case $G_{5}$ ). This is formed by a simple averaging of the top level of the source pyramids. Then, from (3):

$$
\tilde{G}_{k}=\tilde{L}_{k}+\operatorname{EXPAND}\left(G_{k+1}\right)
$$

This process is performed repeatedly to expand each pyramid level. The addition of $\tilde{L}_{k}$ represents the incorporation of detail data at each scale. The complete fused image lies at the bottom of this pyramid, level $G_{0}$. This process mirrors the decomposition process described in Section 2, and again uses FIFOs to store data between pyramid levels, with twodimensional upsampling occurring as data are read from the FIFOs through insertion of zero value samples.

\section{IMPLEMENTATION RESULTS}

The entire design was implemented using Xilinx System Generator, and mapped to a Virtex-2 XC2VP100 device. Synthesis was carried out using Xilinx's proprietary XST tool, as part of ISE8.1. The resource requirements are shown in Table 1. The large RAM requirement is mostly for the delay lines used in the $2 \mathrm{D}$ convolutions. The design is fully pipelined and capable of producing an output pixel every clock cycle. The maximum clock speed reported by the place and route tools is $31 \mathrm{MHz}$.

As a means of comparing the system's performance with a software implementation, the Matlab Profiler was used to measure the speed of execution of a Matlab implementation of the same algorithm. Processing a single frame of data on a $2.8 \mathrm{GHz}$ Pentium-4 processor with 1GB RAM takes, on average, 1.1 seconds. A comparison of the performance of both FPGA and PC based versions of the algorithm is shown in Table 2.

Note that although the maximum reported clock speed is $31 \mathrm{MHz}$, a speed of $10 \mathrm{MHz}$ would allow 8-bit greyscale VGA video at 30fps to be processed in real-time, with a latency of $<50 \mathrm{~ms}$. Sample images have been produced for comparison and are shown in Fig. 3. The source images are from a TV camera and thermal (IR) camera respectively, and are pre-registered. Both source images accentuate different features about the scene being observed. The composite image contains the significant details from both source images. 


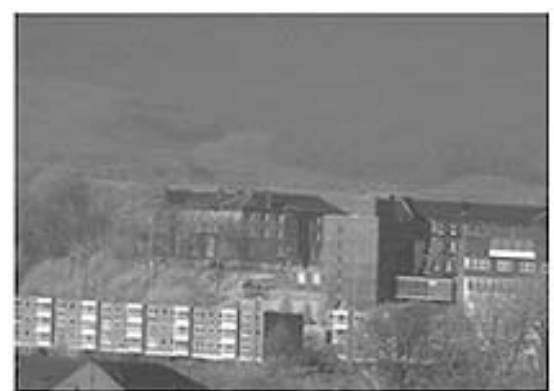

TV humes

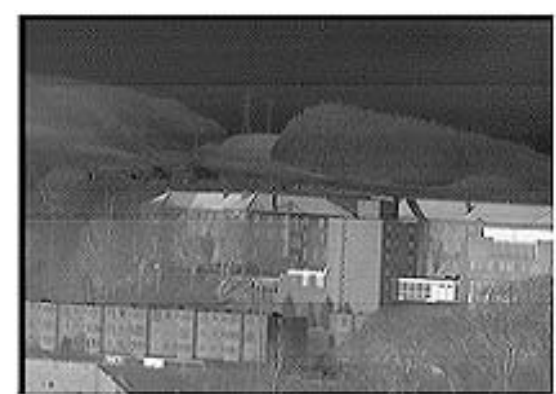

IR Yrage

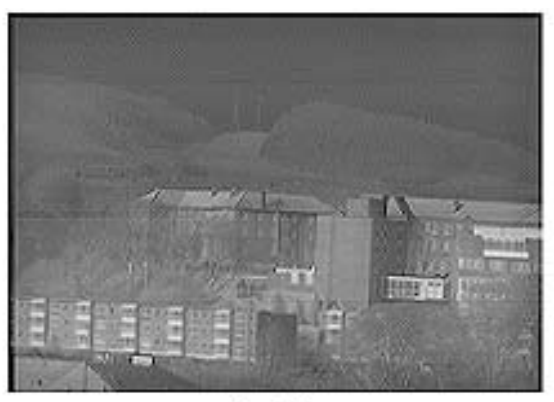

Fused Irapes

Fig. 3. Example of source and fused images.

Table 1. FPGA resource requirements

\begin{tabular}{l|c|c|c} 
Resource & Used & Available & \% of XC2VP100 \\
\hline \hline Slices & 13,287 & 44,096 & 30 \\
\hline 4-input LUTs & 24,533 & 88,192 & 27 \\
\hline Slice FFs & 5,784 & 88,192 & 6 \\
\hline Block RAMS & 430 & 444 & 96
\end{tabular}

Table 2. Performance compared to PC implementation

\begin{tabular}{l|c} 
FPGA Maximum Clock Frequency & $31 \mathrm{MHz}$ \\
\hline FPGA Maximum Frame Rate (8-bit, VGA) & $101 \mathrm{fps}$ \\
\hline $\begin{array}{l}\text { PC/Matlab Frame Rate (2.8GHz P4, 1GB } \\
\text { RAM) }\end{array}$ & $0.91 \mathrm{fps}$ \\
\hline Speedup & $111 \mathrm{x}$
\end{tabular}

\section{CONCLUSION}

A complete implementation of pattern-selective image fusion has been presented that utilises aspects of FPGA technology to enable dual video streams to be processed in realtime. Four levels of pyramidal decomposition, with four separate gradient operators, can all run on a single device with no requirement for off-chip memory. The modular nature of the design means that pyramids with less, or more, levels could be implemented without major modification. Use of an FPGA has enabled a design that can process images at a rate over 100 times faster than a similar PC-based implementation.

\section{ACKNOWLEDGEMENTS}

The authors would like to thank Thales Optronics Ltd. and the Engineering and Physical Sciences Research Council (EPSRC) for their support under the Engineering Doctorate programme.

\section{REFERENCES}

[1] P. K. Varshney, "Multisensor data fusion," Electronics and Communication Engineering Journal, vol. 9, no. 6, pp. 245253, Dec. 1997.

[2] Z. Xue, R. S. Blum, and Y. Li, "Fusion of visual and IR images for concealed weapon detection," in Proc. 5th Int. Conf. Information Fusion, vol. 1, Sept. 2002.

[3] E. H. Adelson, C. H. Anderson, J. R. Bergen, P. J. Burt, and J. M. Ogden, "Pyramid methods in image processing," $R C A$ Engineer, vol. 29, no. 6, Nov. 1984.

[4] P. J. Burt, "A gradient pyramid-basis for pattern-selective image fusion," in Proc. Society for Information Display Conf., 1992.

[5] P. J. Burt and R. J. Kolczynski, "Enhanced image capture through fusion," in Proc. 4th Int. Conf. Computer Vision, May 1993, pp. 173-182.

[6] V. S. Petrović and C. S. Xydeas, "Gradient based multiresolution image fusion," IEEE Trans. Image Processing, vol. 13, no. 2, pp. 228-237, Feb. 2004.

[7] P. Burt and E. Adelson, "The Laplacian pyramid as a compact image code," IEEE Trans. Commun., vol. 31, no. 4, pp. 532540, Apr. 1983.

[8] G. S. van der Wal and P. J. Burt, "A VLSI pyramid chip for multiresolution image analysis," Int. Jour. Computer Vision, vol. 8, no. 3, pp. 177-189, 1992.

[9] C. H. Anderson, "Filter-subtract-decimate hierarchical pyramid signal analyzing and synthesizing technique," U.S. Patent 4718 104, 1987.

[10] A. L. Abbot, P. M. Athanas, L. Chen, and R. L. Elliot, "Finding lines and building pyramids with Splash-2," in Proc. IEEE Workshop on FPGAs for custom computing machines, Apr. 1994, pp. 155-163.

[11] Z. Zhang and R. S. Blum, "A categorization of multiscaledecomposition-based image fusion schemes with a performance study for a digital camera," Proc. IEEE, vol. 87, no. 8, pp. 177-189, Aug. 1999. 